\title{
Long-term results of ablation for isolated atrial fibrillation through a right minithoracotomy: Toward a rational revision of treatment protocols
}

\author{
Giuseppe Nasso, MD, ${ }^{\mathrm{a}}$ Raffaele Bonifazi, MD, ${ }^{\mathrm{a}}$ Armando Del Prete, MD, ${ }^{\mathrm{b}}$ Giuseppe Del Prete, MD, \\ Vincenzo Lopriore, $\mathrm{MD},{ }^{\mathrm{c}}$ Francesco Bartolomucci, $\mathrm{MD},{ }^{\mathrm{d}}$ Antonio Maria Calafiore, $\mathrm{MD},{ }^{\mathrm{e}}$ and \\ Giuseppe Speziale, $\mathrm{MD}^{\mathrm{a}}$
}

\begin{abstract}
Objective: Despite continued technical improvements, results of transcatheter radiofrequency ablation of atrial fibrillation may be suboptimal in some patient subgroups. Short-term follow-up of minimally invasive epicardial ablation of isolated atrial fibrillation has been encouraging.
\end{abstract}

\begin{abstract}
Methods: One hundred four patients with drug-refractory isolated atrial fibrillation underwent minimally invasive surgical ablation through right 3- to 4- $\mathrm{cm}$ minithoracotomy by isolation of pulmonary veins and were followed-up for an average of 17 months. Previous failed transcatheter ablation was not a criterion for this procedure. Antiarrhythmic drugs were continued until postoperative month 6, despite demonstration of stable sinus rhythm.
\end{abstract}

\begin{abstract}
Results: The procedure was confirmed to be safe ( 1 case of procedure-related morbidity, no operative deaths) and effective (89\% overall freedom from recurrent arrhythmia at follow-up, $96 \%$ freedom from paroxysmal atrial fibrillation, $80 \%$ freedom from persisting type atrial fibrillation). Results tended to improve with the expansion of the surgical experience. Cox hazard regression and Kaplan-Meier analysis identified persisting type atrial fibrillation and enlarged left atrium as the major predictors of recurrent atrial fibrillation at follow-up. Health-related quality of life was confirmed to be improved at the end of the follow-up relative to baseline in most Medical Outcomes Study 36-Item Short-Form Health Survey domains.
\end{abstract}

Conclusions: Minimally invasive epicardial ablation of isolated atrial fibrillation yields stable, gradually improving results. Earlier surgical referral is justifiable after careful cardiologic work-up. To define the relative roles of minimally invasive ablation and transcatheter ablation, which may be considered in the future as alternative therapies, a randomized trial to compare these procedures is advisable. (J Thorac Cardiovasc Surg 2011;142:e41-6)

Atrial fibrillation (AF) is among the most common rhythm disturbances in adults. This arrhythmia can lead to important cardiovascular morbidity and mortality, mainly from thromboembolism. ${ }^{1}$ Reduction in health-related quality of life and increases in social expenditures also ensue, even for patients with isolated AF. The rate of failure of medical rhythm control therapy may be as high as $85 \%$ at 2 years. ${ }^{2}$ Transcatheter radiofrequency ablation has been recognized as an effective second-line treatment to obtain rhythm control. ${ }^{3}$ Nonetheless, despite application of state-of-the-art

\footnotetext{
From the Division of Cardiac Surgery, ${ }^{\mathrm{a}}$ GVM Care \& Research, Bari, Italy; the Division of Cardiology, ${ }^{\mathrm{b}}$ San Carlo Hospital, Potenza, Italy; Division of Cardiology, ${ }^{\mathrm{c}}$ San Giacomo Hospital, Monopoli, Italy; the Division of Cardiology, ${ }^{\mathrm{d}}$ L. Bonomo Hospital, Andria, Italy; and the Division of Cardiac Surgery, ${ }^{\mathrm{e}}$ Prince Sultan Cardiac Centre, Riyadh, Saudi Arabia.

Disclosures: Authors have nothing to disclose with regard to commercial support.

Received for publication Nov 5, 2010; revisions received Feb 21, 2011; accepted for publication April 8, 2011; available ahead of print May 16, 2011.

Address for reprints: Giuseppe Nasso, MD, Department of Cardiac Surgery, Anthea

Hospital, GVM Care \& Research, Via Camillo Rosalba 35-37, 70124 Bari, Italy

(E-mail: gnasso@libero.it).

$0022-5223 / \$ 36.00$

Copyright (c) 2011 by The American Association for Thoracic Surgery

doi:10.1016/j.jtcvs.2011.04.009
}

technologies, the recurrence rate of AF may be still considerable (about $30 \%$ at 30 months). ${ }^{4}$

Currently, the surgical epicardial ablation of isolated, drug-refractory AF is possible through minimally invasive access and with the heart beating. The safety of this technique and encouraging short-term results were reported from our initial experience, including a $13 \%$ rate of recurrence at 6 months. ${ }^{5} \mathrm{AF}$ is an insidious disease, however, and may recur over time. Therefore longer follow-up periods and a greater number of patients are required to address adequately the reliability of any ablation technique. This article outlines the results of the minimally invasive ablation of isolated $\mathrm{AF}$ at midterm follow-up and discusses the potential implications for the current treatment algorithms for isolated $\mathrm{AF}$.

\section{MATERIALS AND METHODS \\ Patient Selection}

The program of epicardial minimally invasive ablation of isolated AF was started at our institution in June 2008. A total of 104 patients underwent this treatment and were enrolled in this investigation. Patients were scheduled for surgery on the basis of the following criteria: (1) recurrent 


\section{Abbreviations and Acronyms \\ $\mathrm{AF} \quad=$ atrial fibrillation \\ SR = sinus rhythm}

episodes of paroxysmal or persisting isolated AF refractory to the maximal tolerated doses of class IC or III antiarrhythmic agents, alone or in combination, and (2) at least 1 failed electrical or pharmacologic cardioversion attempt during the 6 months preceding the surgical evaluation. At preoperative echocardiography, the left atrial diameter was assessed in the parasternal long-axis view and indexed to the body surface area. In the second half of our experience, significantly but not excessively increased left atrial dimension was not considered an exclusion criterion for the procedure; however, left atrial dimension indexed to body surface area exceeding $35 \mathrm{~mm} / \mathrm{m}^{2}$ was considered an exclusion criterion for the procedure in the entire study population. All enrolled patients with indexed left atrial sizes between 22 and $35 \mathrm{~mm} / \mathrm{m}^{2}$ were considered to have an enlarged left atrium. AF was considered paroxysmal when self-terminating and lasting less than 7 days. Persisting AF was diagnosed when AF lasted more than 7 days but less than 6 months or when it had to be terminated by direct-current or pharmacologic cardioversion.

\section{Surgical Technique}

Oral anticoagulation therapy was withdrawn 3 days before the procedure, and the patients were put on a regimen of enoxaparin sodium at 4000 IU twice daily until the evening before surgery. The preoperative anticoagulation regimen was resumed on the day after surgery. The operative technique has been already described in a previous publication. ${ }^{5}$ Briefly, with the patient under general anesthesia, a 3- or 4-cm right minithoracotomy was performed at the $3 \mathrm{rd}$ intercostal space, and a soft-tissue retractor was applied. Transesophageal echocardiography was performed before commencement of the procedure to rule out intra-atrial thrombus. To ensure lack of thrombus, we submitted patients to isolated AF surgery after a conventional period of at least 3 weeks of oral anticoagulant therapy. ${ }^{6}$ It was generally feasible to reach the pericardium while keeping both lungs inflated by means of a gentle traction to the middle lobe of the right lung. Collapse of the right lung could be achieved whenever required to ameliorate the exposure. Under direct vision but with the help of thoracoscopic camera, the pericardium was incised parallel to the right phrenic nerve and the oblique sinus was entered by blunt dissection. The COBRA Adhere XL System (Estech, San Ramon, Calif) was used in all cases. This device is designed to obtain electrical isolation of the pulmonary veins by radiofrequency ablation of the atrial myocardium in an entirely off-pump procedure. An introducer equipped with a magnetic tip was pushed into the transverse sinus until the left atrial appendage was passed. A second introducer was pushed into the oblique sinus until its tip joined the first introducer. A loop was then formed, and at this stage care was taken to avoid inadvertent lesion of the left atrial appendage. The ablation probe was guided into position, and its branches were tightened so that the 4 pulmonary veins were encircled. The device used suction to stabilize the contact with the myocardium and achieve uniform energy delivery. A circular box lesion was created with radiofrequency energy. Different patterns of energy delivery are available with the device used; in our experience, ablation was performed more commonly by 2 energy applications lasting 150 seconds each. These were followed by a 60 -second application after the probe was moved circumferentially, to achieve complete closure of the box lesion. After the probe was withdrawn, efficacy assessment was usually done by measurement of conduction across the lesion. Conduction block was evaluated with pacing from the pulmonary veins (exit block). Complete block was considered to be indicative of effective electrical isolation. The operative field was inspected, and the chest was closed.

\section{Follow-up}

The patients were transferred to the intensive care unit, where electrical cardioversion could be performed in the early postoperative period whenever needed. The patients were scheduled for regular follow-up visits at 3 and 6 months after discharge from the hospital and every 6 months thereafter. Visits consisted of physical examination, New York Heart Association functional classification, surface electrocardiogram, 24-hour electrocardiographic monitoring, and transthoracic echocardiography. The echocardiographic examination included pulsed Doppler analysis of the transmitral flow (the presence of a filling A wave with a peak flow velocity of $0.4 \mathrm{~m} / \mathrm{s}$ or more was considered indicative of adequate atrial transport function). Telephone interviews were also performed on a monthly basis to enquire as to each patient's status. Patients were also instructed to contact our center in the event of any symptoms. After such contact, a patient was admitted for an unscheduled visit, including 24-hour electrocardiographic tracings. Health-related quality of life was assessed before surgery and at any follow-up visit by administration of the Medical Outcomes Study 36-Item Short-Form Health Survey. ${ }^{7}$ This questionnaire provides a score for each of the following domains: physical activity, role limitation, physical pain, general health, vitality, social activity, and mental health.

According to our protocol, all patients were discharged on a regimen of both oral amiodarone (or flecainide acetate) and warfarin sodium despite demonstration of stable sinus rhythm (SR). Dismal clinical results and unacceptably high rates of AF recurrence may follow if antiarrhythmic medications are withheld immediately after successful ablation. ${ }^{8}$ After demonstration of stable SR at both the 3- and 6-month visits, amiodarone (or other antiarrhythmic drug) was withdrawn. For all patients who had the antiarrhythmic drugs suspended, additional 24-hour electrocardiographic monitoring was scheduled at 1 and 3 months after drug withdrawal. Whenever stable SR was found to be maintained 3 months later on, oral anticoagulation was withdrawn as well. For the purposes of this study, thromboembolic events were defined as radiologic demonstration of a new ischemic area within the brain (ischemic stroke) associated with a new focal neurologic deficit or coma.

\section{Study End Points}

Study end points were as follows:

1. One-year clinical results of rate of recurrence of AF, freedom from antiarrhythmic medications, freedom from oral anticoagulant drugs, freedom from thromboembolic events, and health-related quality-of-life.

2. Left atrial transport function at 1-year follow-up (pulsed Doppler examination).

3. Factors associated with AF recurrence during follow-up.

\section{Data Management and Statistical Analysis}

Individual patients' data were prospectively collected with preformatted data sheets and included in an electronic database, which was periodically checked for errors and omissions. Data were processed with SPSS software version 13.0 (SPSS Inc, an IBM Company, Chicago, Ill). Continuous variables are presented as mean $\pm \mathrm{SD}$, and categorical variables are presented as percentages. Intergroup comparisons were performed with the 2-tailed Student $t$ test (continuous variables) or the $\chi^{2}$ test (categorical variables) for unpaired data. Time-to-event analysis (Cox proportional hazards regression) was used to identify the predictors of AF recurrence during the follow-up. The assumptions of Cox regression were checked and met, and the model was successfully tested. The variables entered in the model as potential explanatory variables were the following: age, sex, type of $\mathrm{AF}$ (persisting or paroxysmal), left atrial dimension, previous electrical cardioversion or transcatheter ablation, pattern of radiofrequency ablation applications delivered, preoperative New York Heart Association functional status, left ventricular ejection fraction, diabetes mellitus, arterial hypertension, and class of antiarrhythmic drugs used. For the purposes of Cox hazards regression, the left atrial diameter was entered as a continuous 
variable. Meanwhile, for the construction of stratified Kaplan-Meier curves, the left atrial dimension was managed as a dichotomous variable. In such instance, an indexed left atrial size of at least $22 \mathrm{~mm} / \mathrm{m}^{2}$ was considered indicative of an enlarged left atrium. Recurrent AF-free survival was analyzed according to the Kaplan-Meier method, and corresponding survival curves were built. Comparisons of survival estimates for different patient strata were performed with the log-rank statistic.

\section{RESULTS}

\section{Operative Results}

The baseline characteristics of the overall study population are reported in Table 1. A total of 104 patients were included in this investigation. Intraoperative transesophageal echocardiography could not demonstrate the presence of intra-atrial thrombus in any patient. Mean operative time was $82 \pm 33$ minutes. In the overall population, we had no operative deaths and 3 cases of periprocedural complications. Intraoperative rupture of the left atrial appendage occurred in 1 case during the earliest phase of our surgical experience; emergency sternotomy was required for repair. In 1 patient, hemorrhagic stroke with hemiplegia occurred 4 days after surgery; this patient was receiving long-term anticoagulant therapy and had 2 previous episodes of intracranial hemorrhage before being submitted to arrhythmia surgery. We therefore considered this morbidity episode not to be procedure related. The third patient had a transient ischemic attack in the early postoperative period; no thrombus had been localized within the left atrium or left appendage. Both patients were in SR at the time of morbidity. Twenty patients $(19.5 \%)$ were subjected to electrical cardioversion before hospital discharge as a result of persisting AF or supraventricular tachycardia. No further instances of perioperative morbidity were recorded. All patients but 1 were discharged in SR and on a regimen of antiarrhythmic drugs (amiodarone or flecainide).

\section{Follow-up Results}

The average follow-up was $17.1 \pm 5.8$ months (range, 2-19 months). Follow-up was $100 \%$ complete. The results obtained in the overall study cohort are summarized in Table 2. During the follow-up, 2 patients died of noncardiac related causes (trauma in 1 case and malignant neo-

TABLE 1. Baseline characteristics of the overall study population $(\mathbf{n}=\mathbf{1 0 4})$

\begin{tabular}{lc}
\hline Age $(\mathrm{y}$, mean $\pm \mathrm{SD})$ & $63.9 \pm 8$ \\
Sex (male/female ratio) & $39: 65$ \\
Left atrial dimension $\left(\mathrm{mm} / \mathrm{m}^{2}\right.$, mean $\left.\pm \mathrm{SD}\right)$ & $21.3 \pm 3$ \\
Diabetes mellitus (no.) & $21(20.2 \%)$ \\
Hypertension (no.) & $54(51.9 \%)$ \\
Previous electrical cardioversion (no.) & $36(34.6 \%)$ \\
Previous transcatheter ablation (no.) & $24(23.1 \%)$ \\
Class I antiarrhythmic drugs (no.) & $39(37.5 \%)$ \\
Class II antiarrhythmic drugs (no.) & $8(7.7 \%)$ \\
Class III antiarrhythmic drugs (no.) & $68(65.4 \%)$ \\
\hline
\end{tabular}

TABLE 2. Follow-up results in the overall study population $(n=104)$ Intensive care unit stay $(\mathrm{h}$, mean $\pm \mathrm{SD}) \quad 18.1 \pm 4.7$ Operative morbidity (any, no.) $3(2.9 \%)$

Recurrent atrial fibrillation (no.) $11(10.6 \%)$

Freedom from antiarrhythmic drugs (no.)

$53(51 \%)$

Freedom from oral anticoagulant therapy (no.) $41(39.4 \%)$

Thromboembolic events (no.) $1(0.9 \%)$

No evidence of atrial transport function* (no.)

$12(11.5 \%)$

*Impossible to demonstrate filling A wave with peak flow velocity of $0.4 \mathrm{~m} / \mathrm{s}$ or more at pulsed transmitral Doppler.

plasm in the second case). One thromboembolic event was recorded. At the end of the follow-up, we recorded a total of 11 clinical relapses of AF $(89.4 \%$ overall freedom from AF). Two of the 11 relapses occurred in patients who had a preoperative diagnosis of paroxysmal $\mathrm{AF}(P=.02 \mathrm{vs} \mathrm{pa-}$ tients with a diagnosis of persisting AF). Cardioversion was performed during the follow-up in 6 of these patients. Thus the freedoms from recurrent $\mathrm{AF}$ at the end of the follow-up were $96 \%$ among patients with a preoperative diagnosis of paroxysmal AF and $80 \%$ among patients with a preoperative diagnosis of persisting AF. According to the previously described protocol, 41 patients who were in stable SR after the 9th postoperative month had both the antiarrhythmic and the anticoagulant medications withdrawn (freedom from any drug $39.4 \%$ ). An additional 12 patients (11.5\%) stopped taking antiarrhythmic drugs after the 6th postoperative month. All patients who had the antiarrhythmic drugs withdrawn remained in stable SR during all the available follow-up after drug withdrawal (average time $4.1 \pm 0.9$ months). Among the 41 patients who were no longer receiving antiarrhythmic drugs, $8(19 \%)$ independently suspended their pharmacologic therapy at an average of 4.5 postoperative months. As stated previously, none of these patients had recurrent atrial arrhythmia during the available follow-up. Conversely, 5 of the 11 patients who had recurrent $\mathrm{AF}(45 \%)$ independently gave up their antiarrhythmic drugs before reaching the scheduled follow-up reassessment. Pulsed Doppler examination performed at the latest follow-up visit available indicated that 92 of 93 patients in SR $(98.9 \%)$ had signs of atrial function. A-wave velocity in these patients ranged from $0.4 \mathrm{~m} / \mathrm{s}$ to $0.76 \mathrm{~m} / \mathrm{s}$ (average $0.51 \pm 0.2 \mathrm{~m} / \mathrm{s}$ ). The single patient without atrial transport function despite lack of electrocardiographic evidence of AF had a preoperative history of the persisting type of AF and an enlarged left atrium.

Table 3 reports the results of Cox proportional hazards regression. Significant predictors of AF recurrence during the

TABLE 3. Results of Cox proportional hazards regression: Predictors of recurrence and time to recurrence of atrial fibrillation

\begin{tabular}{lrcr}
\hline \multicolumn{1}{c}{ Characteristic } & Wald & Risk ratio & $\boldsymbol{P}$ value \\
\hline Persisting atrial fibrillation & 5.3 & 1.36 & .004 \\
Left atrial dimension & 11.8 & 3.7 & $<.001$ \\
\hline
\end{tabular}




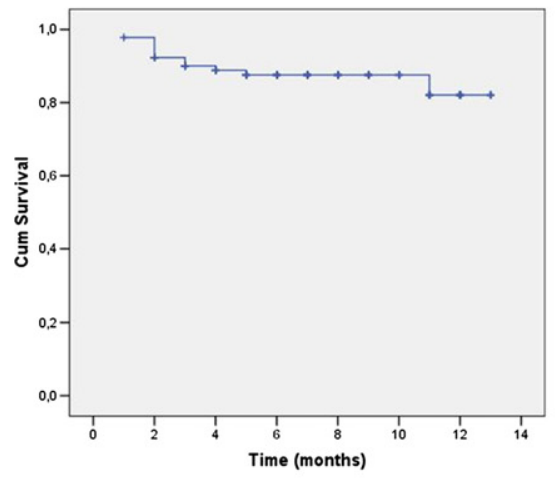

A $\neg$ Survival Function
+ Censored

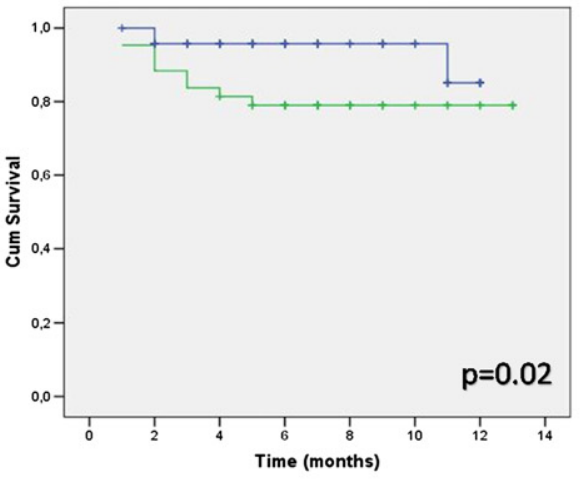

$\neg$ Paroxysmal

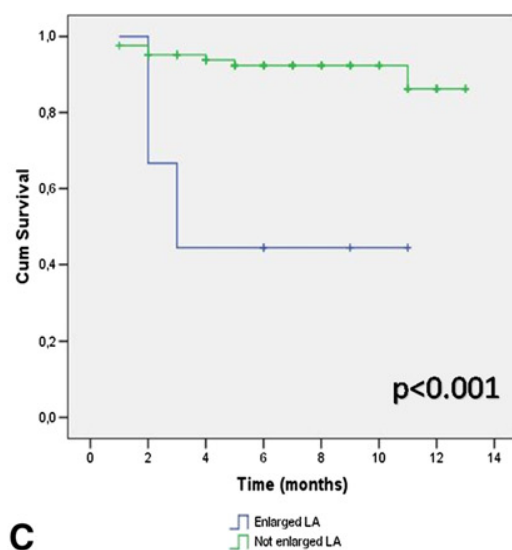

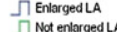

FIGURE 1. Kaplan-Meier survival curves. A, Recurrent atrial fibrillation-free survival in the overall study population. B, Recurrent atrial fibrillation-free survival in patients with preoperative diagnosis of paroxysmal atrial fibrillation (upper line) versus persisting atrial fibrillation (lower line log-rank $P=.02$ ). $\mathrm{C}$, Recurrent atrial fibrillation-free survival in patients with preoperative demonstration of enlarged left atrium (LA, lower line) versus patients without significantly increased left atrial dimension (upper line, log-rank $P<.001$ ). Cum, Cumulative.

follow-up were the persisting type of AF and increased left atrial dimension. Kaplan-Meier analysis indicated that both the persisting type of $\mathrm{AF}$ and enlarged left atrium were significantly associated with a worse AF-free survival (Figure 1). These findings confirm previous data and have potential implications regarding the selection of patients to be submitted to surgery for isolated AF.

In Table 4, we have compared the baseline characteristics and clinical results of the patients operated on during the initial part of our surgical experience (as previously reported $^{5}$ ) with those of the patients who were operated on subsequently. There was no statistically significant difference in terms of AF relapse rate between patients who had an average 6 months of follow-up and those who had a longer average follow-up ( $8.6 \%$ vs $13 \%$, respectively). A trend toward a lower rate of recurrent $\mathrm{AF}$ was observed in the latter group, although the difference did not reach sta-

TABLE 4. Comparison of baseline characteristics, operative characteristics, and follow-up results of patients who underwent minimally invasive ablation of isolated atrial fibrillation in the first experience period $(n=46)$ versus the second experience period $(n=58)$

\begin{tabular}{|c|c|c|c|}
\hline Characteristic & First & Second & $P$ value \\
\hline $\begin{array}{l}\text { Left atrial dimension }\left(\mathrm{mm} / \mathrm{m}^{2} \text {, }\right. \\
\text { mean } \pm \mathrm{SD})\end{array}$ & $21.2 \pm 3$ & $21.4 \pm 3$ & .58 \\
\hline Age $(y$, mean $\pm S D)$ & $63.4 \pm 9$ & $64.1 \pm 9$ & .82 \\
\hline Diabetes mellitus (no.) & $10(21.7 \%)$ & $11(19 \%)$ & .7 \\
\hline Hypertension (no.) & $26(56.5 \%)$ & $28(48.3 \%)$ & .45 \\
\hline $\begin{array}{l}\text { Previous failed } \\
\text { electrocardioversion (no.) }\end{array}$ & $14(30.4 \%)$ & $19(32.7 \%)$ & .9 \\
\hline $\begin{array}{l}\text { Intensive care unit stay } \\
\qquad(\mathrm{h}, \text { mean } \pm \mathrm{SD})\end{array}$ & $18.7 \pm 5.1$ & $17.7 \pm 5$ & .71 \\
\hline Operative morbidity (any, no.) & $2(1.9 \%)$ & 1 & .8 \\
\hline $\begin{array}{l}\text { Recurrent atrial fibrillation at } \\
\text { follow-up (no.) }\end{array}$ & $6(13.04 \%)$ & $5(8.6 \%)$ & .12 \\
\hline
\end{tabular}

tistical significance. This observation, nevertheless, may be clinically significant. Such a concept is strengthened by the comparability of these groups in terms of the baseline factors associated with the AF burden (left atrial dimension, age, diabetes mellitus, hypertension, and left ventricular ejection fraction).

We confirm previous findings that the average 36-Items Short-Form Health Survey-related domains show a statistically significant improvement at the end of the follow-up relative to the baseline value, except for the vitality indices. Particularly, a remarkable amelioration was seen for the following parameters: physical activity $(P<.001)$, role limitation $(P<.001)$, general health $(P<.001)$, social activities $(P<.001)$, and mental health $(P=.02)$.

\section{DISCUSSION}

A therapeutic gap still exists for patients with isolated AF. Despite ongoing research and continued improvements in the treatment protocols and transcatheter radiofrequency ablation technology, the rate of recurrence of AF after second-line treatment (transcatheter ablation) in patients with drug-refractory AF may reach $30 \%{ }^{4}$ Many patients therefore remain exposed to thromboembolic events and need anticoagulation indefinitely. This study was conceived to evaluate the long-term clinical results of the radiofrequency surgical epicardial ablation of isolated AF through minimally invasive access. A device designed for monolateral right minithoracotomy approach and a completely offpump strategy were used. ${ }^{9}$ It has been already reported that such device has a good safety and feasibility profile, and the early results have been encouraging ( $87 \%$ freedom from recurrent $\mathrm{AF}$ at an average of 6 months). ${ }^{5}$ Here we report a continued follow-up (17.1 \pm 5.8 months $)$ and a larger series. The expanding experience led to a reduced rate of procedure-related serious adverse events and to a gradual 
improvement in the rate of clinical success (Table 4). The global rate of freedom from recurrent $\mathrm{AF}$ was $89.4 \%$ at the end of the follow-up. This is reflected by the finding that the benefits in terms of the quality-of-life indices are consolidated with time.

In this series, we also analyzed the first cohort of patients who had their antiarrhythmic and anticoagulant drug regimen withdrawn after achievement of stable SR during the follow-up. About $51 \%$ of all patients were no longer receiving antiarrhythmic drugs at the end of the follow-up. All these patients remained in stable SR after the withdrawal of the antiarrhythmics (average follow-up available after drug withdrawal, $4.1 \pm 0.9$ months), which testifies to the stability with time of the results of this procedure. Most clinical recurrences of arrhythmia apparently occur within a shorter period after surgery (average, 3.4 postoperative months in our series). Interestingly, this resembles previous evidence obtained for transcatheter ablation. ${ }^{4}$ Overall, improved health-related quality of life and freedom from any drugs represent the true therapeutic goal to be achieved for these patients.

The operation described here holds the advantages of any minimally invasive surgery: reduced morbidity, shortened hospitalization, and minimized scar formation. The ablation procedure is entirely off pump. This may consolidate the clinical results in terms of early freedom from arrhythmia, because postpump systemic inflammatory reaction is a known independent trigger of $\mathrm{AF} .{ }^{10}$ The ablation procedure that we used is, however, confined to the left atrium. Reduced exposure of the cardiac structures is the price we pay to achieve minimal invasiveness and a fully off-pump treatment. In a meta-analysis, it was reported that adding a set of right atrial lesions to the isolation of the pulmonary veins could confer superior results in terms of late arrhythmia recurrence relative to the ablation patterns limited to the left atrium. ${ }^{11}$ This meritorious work is limited, however, by the inclusion of several retrospective trials published in the 1995 to 2005 period. More recently, randomized studies have been conducted with novel ablation equipment and have underlined that the clinical results (in terms of maintenance of SR and freedom from thromboembolic events) were comparable between the biatrial and left atrial procedures. ${ }^{8,12,13}$ Such conclusions corroborate the practice of the minimally invasive radiofrequency isolation of the pulmonary veins, provided that adequate lesions can be obtained (transmurality and lesion continuity are pivotal requirements). Electrophysiologic data indicate that $\mathrm{AF}$ generally has a biatrial pathophysiologic substrate in patients with isolated arrhythmia. ${ }^{14}$ Nonetheless, our data suggest that the electrical isolation of the pulmonary veins is effective in reestablishing SR in most cases of isolated AF. Electrophysiologic studies were not available in most of our cases. The literature uniformly indicates that increased left atrial dimension is the main predictor of AF re- currence, and our results for patients with indexed left atrial sizes between 22 and $35 \mathrm{~mm} / \mathrm{m}^{2}$ (Cox regression and Kaplan-Meier analysis) confirm this concept. Atrial dilatation is considered a hallmark of established myocardial derangements, even at the cellular level; such alterations are not reversible and perpetuate the arrhythmia even in patients with isolated AF. At this stage, any ablative intervention has lesser likelihood of success both in patients with concomitant organic heart disease and in patients with isolated AF. This is in agreement with the finding that less optimal results are obtained at follow-up in patients with persisting rather than paroxysmal AF (Cox regression and Kaplan-Meier analysis). ${ }^{15}$ Thus the degree of atrial derangement should be probably considered the major pathophysiologic marker of disease progression. The clinician may establish decision making and surgical timing according to such a marker. Ablation is most effective before excessive left atrial alterations have set in, and earlier referral may therefore be recommended. We encourage the development of larger investigations with comparison of patients with paroxysmal versus persisting $\mathrm{AF}$ and comparisons of patients belonging to different strata according to left atrial dimension. Consideration of minimally invasive ablation for patients with an indexed left atrial size greater than $35 \mathrm{~mm} / \mathrm{m}^{2}$ should be evaluated. Ultimately, such parameters (left atrial size class and AF type) should be regarded as potential tools to refine the surgical timing.

A good concordance existed between electrocardiographic documentation of stable SR at follow-up and demonstration of atrial contraction. Nonetheless, our data confirm that a $100 \%$ concordance may not be achieved. The clinician thus should probably include echocardiographic assessment of the atrial function in all patients followed up after AF ablation, mainly if enlarged left atrium and persisting type of AF coexist. Anticoagulant therapy for prevention of ischemic stroke may be then considered.

Follow-up for arrhythmia recurrence was conducted with 24-hour electrocardiographic monitoring in this study. The unavailability of data from 72-hour tracings and internal loop recorder represents a limitation of this investigation. Nonetheless, telephonic monitoring, patient education to report any symptoms promptly, and liberal use of unscheduled visits and 24-hour electrocardiographic tracings are likely to minimize arrhythmia underdetection during follow-up.

In conclusion, this study supports the contention that the results of minimally invasive ablation of drug-refractory isolated AF by radiofrequency isolation of the pulmonary veins are reliable with time (1-year average follow-up) and that stable SR can be achieved with this technique in most cases. The results tend to improve with the expansion of the surgical experience. The rate of withdrawal of antiarrhythmic and anticoagulant drugs is encouraging, although the precise requirement and duration of the postoperative 
antiarrhythmic regimen remain to be ascertained. Nonetheless, it is remarkable that $45 \%$ of the patients undergoing AF relapse independently suspended the antiarrhythmic medications. This therapeutic option may be considered for all patients with isolated, drug-refractory AF and previous failed cardioversion. The selection of candidates can be enhanced, bearing in mind that increased left atrial size and persisting type of AF is closely associated with late AF recurrence. Earlier referral to surgery of patients with drugrefractory isolated AF is therefore justifiable after a careful cardiologic workup. The epicardial, minimally invasive, off-pump ablation and the transcatheter ablation may be regarded in the future as alternative therapies; minimally invasive surgery may be also considered as the first-line treatment for selected patients with isolated AF. Nevertheless, a randomized trial should now be designed to compare directly these 2 approaches, in view of the very good results, safety, and consolidated experience with minimally invasive epicardial surgery.

\section{References}

1. Ezekowitz M, Netrebko P. Anticoagulation in management of atrial fibrillation. Curr Opin Cardiol. 2003;18:26-31.

2. Lundström T, Rydén L. Chronic atrial fibrillation. Long-tem results of direct current cardioversion. Acta Med Scand. 1988;223:53-9.

3. Terasawa T, Balk E, Chung M, Garlitski AC, Alsheikh-Ali AA, Lau J, et al. Systematic review: comparative effectiveness of radiofrequency catheter ablation for atrial fibrillation. Ann Intern Med. 2009;151:191-202.

4. Miyazaki S, Kuwahara T, Kobori A, Takahashi Y, Takei A, Sato A, et al. Longterm clinical outcome of extensive pulmonary vein isolation-based catheter ablation therapy in patients with paroxysmal and persistent atrial fibrillation. Heart. 2011;97:668-73.
5. Speziale G, Bonifazi R, Nasso G, Bartolomucci F, Caldarola P, Fattouch K, et al. Minimally invasive radiofrequency ablation of lone atrial fibrillation by monolateral right minithoracotomy: operative and early follow-up results. Ann Thorac Surg. 2010;90:161-7.

6. Fuster V, Rydén L, Cannom D, Crijns HJ, Curtis AB, Ellenbogen KA, et al. ACC/ AHA/ESC 2006 Guidelines for the Management of Patients with Atrial Fibrillation: a report of the American College of Cardiology/American Heart Association Task Force on Practice Guidelines and the European Society of Cardiology Committee for Practice Guidelines (Writing Committee to Revise the 2001 Guidelines for the Management of Patients With Atrial Fibrillation). Circulation. 2006;114:e257-354.

7. Aaronson N, Acquadro C, Alonso J, Apolone G, Bucquet D, Bullinger M, et al. International Quality of Life Assessment (IQOLA) Project. Qual Life Res. 1992; $1: 349-51$

8. Srivastava V, Kumar S, Javali S, Rajesh TR, Pai V, Khandekar J, et al. Efficacy of three different ablative procedures to treat atrial fibrillation in patients with valvular heart disease: a randomised trial. Heart Lung Circ. 2008;17:232-40.

9. Bevilacqua S, Gasbarri T, Cerillo AG, Mariani M, Murzi M, Nannini T, et al. A new vacuum-assisted probe for minimally invasive radiofrequency ablation. Ann Thorac Surg. 2009;88:1317-21.

10. Anselmi A, Possati G, Gaudino M. Postoperative inflammatory reaction and atrial fibrillation: simple correlation or causation? Ann Thorac Surg. 2009;88:326-33.

11. Barnett S, Ad N. Surgical ablation as treatment for the elimination of atrial fibrillation: a meta-analysis. J Thorac Cardiovasc Surg. 2006;131:11029-35.

12. Wang J, Meng X, Li H, Cui Y, Han J, Xu C. Prospective randomized comparison of left atrial and biatrial radiofrequency ablation in the treatment of atrial fibrillation. Eur J Cardiothorac Surg. 2009;35:116-22.

13. Albrecht A, Kalil RA, Schuch L, Abrahão R, Sant'Anna JR, de Lima G, et al. Randomized study of surgical isolation of the pulmonary veins for correction of permanent atrial fibrillation associated with mitral valve disease. $J$ Thorac Cardiovasc Surg. 2009;138:454-9.

14. Rostock T, Steven D, Hoffman B, Servatius H, Drewitz I, Sydow K, et al. Chronic atrial fibrillation is a biatrial arrhythmia: data from catheter ablation of chronic atrial fibrillation aiming arrhythmia termination using a sequential ablation approach. Circ Arrhythm Electrophysiol. 2008;1:344-53.

15. Bhargava M, Di Biase L, Mohanty P, Prasad S, Martin DO, WilliamsAndrews M, et al. Impact of atrial fibrillation and repeat catheter ablation on long-term freedom from atrial fibrillation: results from a multicenter study. Heart Rhythm. 2009;6:1403-12. 